\title{
Study of Forebody Injection and Mixing with Application to Hypervelocity Airbreathing Propulsion
}

\author{
Erik Axdahl* and Ajay Kumar ${ }^{\dagger}$ \\ NASA Langley Research Center, Hampton, VA 23681 \\ Alan Wilhite $\ddagger$ \\ National Institute of Aerospace, Hampton, VA 23666
}

The use of premixed, shock-induced combustion in the context of a hypervelocity, airbreathing vehicle requires effective injection and mixing of hydrogen fuel and air on the vehicle forebody. Three dimensional computational simulations of fuel injection and mixing from flush-wall and modified ramp and strut injectors are reported in this study. A well-established code, VULCAN, is used to conduct nonreacting, viscous, turbulent simulations on a flat plate at conditions relevant to a Mach 12 flight vehicle forebody. In comparing results of various fuel injection strategies, it is found that strut injection provides the greatest balance of performance between mixing efficiency and stream thrust potential.

\section{Nomenclature}

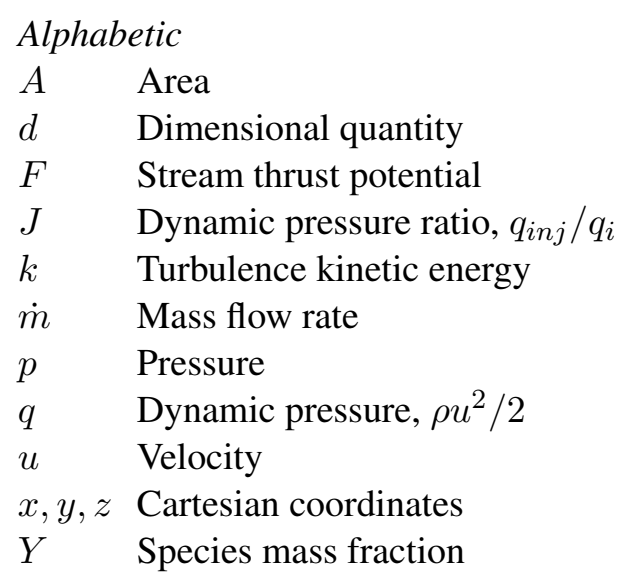

\section{Greek \\ $\beta \quad$ Ramp sweep angle \\ $\varepsilon \quad$ Turbulence dissipation \\ $\eta_{m} \quad$ Mixing efficiency \\ $\omega \quad$ Ratio of turbulence dissipation to kinetic energy, $\varepsilon / k$}

${ }^{*}$ Aerospace Engineer, Hypersonic Airbreathing Propulsion Branch, MS168, Member AIAA

${ }^{\dagger}$ Director, Systems Analysis and Concepts Directorate, MS449, Fellow AIAA

${ }^{\ddagger}$ Professor, Department of Aerospace Engineering, Georgia Institute of Technology, Associate Fellow AIAA 
$\phi \quad$ Equivalence ratio

$\rho \quad$ Density

\section{Subscript}

1,2 Major and minor dimensions, respectively

exp Expanded condition

$R \quad$ Least-available reactant

$S \quad$ Stoichiometric

\section{Introduction}

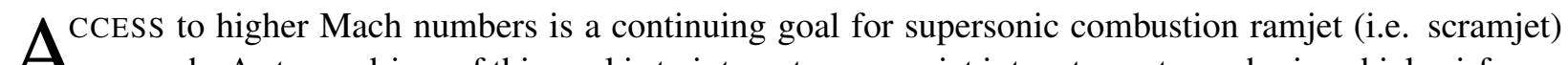
A research. A strong driver of this goal is to integrate a scramjet into a transatmospheric vehicle airframe to improve single- or two-stage-to-orbit performance to low earth orbit. Beyond Mach 10, however, scramjets are projected to experience significant loss in efficiency approaching that of a conventional rocket. One reason for this is the diminishing time with increasing Mach number to inject and mix fuel with supersonic air and complete the combustion process.

The state of the art for propulsion in the hypervelocity regime (i.e. above Mach 10) is advanced by taking advantage of premixed, shock-induced combustion (PMSIC). An example of an engine using this technique is the shock-induced combustion ramjet (i.e. shcramjet). In a shcramjet, fuel is injected on the vehicle forebody where it mixes with the captured air stream, after which the fuel-air mixture enters the inletcombustor where a shock wave induces ignition. Since the mixing occurs on the forebody, the combustor is significantly shortened allowing for lower weight and a reduced heat load. A reduced heat load will mitigate or eliminate the amount of excess fueling (i.e. fueling above the design flow rate to increase the fuel cooling capcity) required to cool the engine, thereby increasing the specific impulse of the shcramjet over the scramjet.

PMSIC technical challenges that must be overcome are indicated in Figure 1. First is the ability to achieve uniform mixing of the fuel and air along the forebody without spillage around of the cowl lip. Second is the prevention of autoignition of the fuel-air mixture as it enters the hot boundary layer of the forebody. Third is the issue of shock wave stability at the entrance to the combustor ${ }^{1}$. Consequences of not meeting these technical challenges include combustion instability due to nonuniform mixing, adverse pressure drag on the forebody due to premature combustion, and loss of engine performance due to fuel spillage.

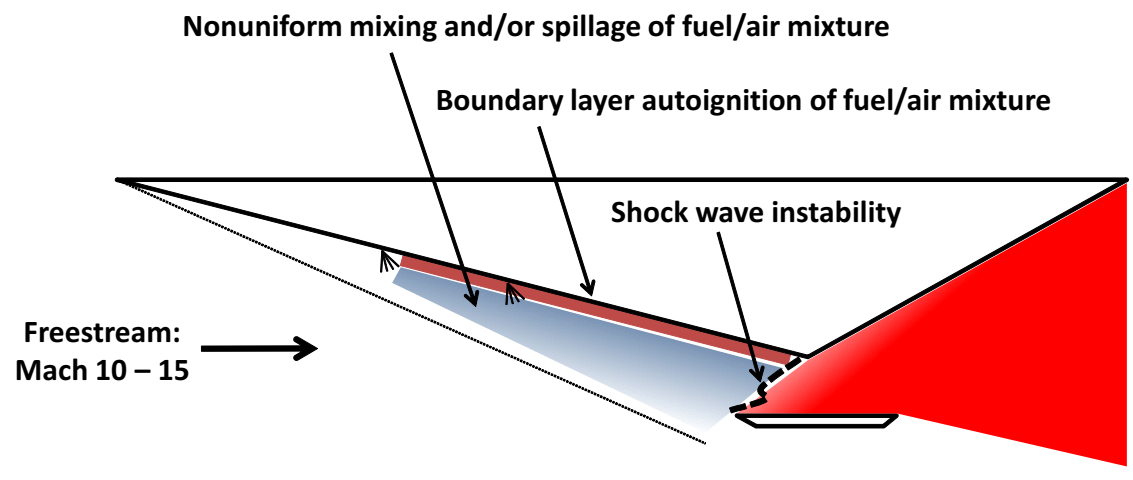

Figure 1. Technical challenges experienced by shcramjet concepts are shown. 
This paper will focus primarily on understanding the physics of mixing at high Mach numbers through the numerical simulation of flush-wall and off-wall injector geometries. Stoichiometric hydrogen injection will be studied in flat plate flow representative of forebody conditions relevant to a Mach 12 vehicle traveling along a typical dynamic pressure trajectory. First, flush-wall injection will be studied with various injector nozzle exit shapes to characterize mixing efficiency and losses for each geometry. Next, ramp and strut injector geometries will be evaluated with the same metrics as the wall injection cases. All injectors will be assumed to be in a symmetric array in order to include the effect of interaction between neighboring injectors.

\section{Previous Work}

Much of the literature over the past sixty years on fuel injection research for scramjet combustion has been at Mach numbers representative of mid-range hypersonic flight (Mach 5 - 8). Of these, most numerical and experimental studies have been conducted at conditions relevant to the combustor as typical fuel injection schemes begin at the transition from the isolator to the combustor. Therefore, most studies have taken place around Mach 2 - 3 in duct flow. In this speed regime, significant experimental attention has been paid toward the evaluation of perpendicular wall injection, ${ }^{2}$ near-parallel injection concepts (e.g. swept ramp injectors), ${ }^{3,4}$ transpiration/effusive injection cooling for transverse injection, ${ }^{5}$ and the effect of fuel injector nozzle geometry on mixing characteristics ${ }^{6-8}$. The use of cavities for injection and flameholding has also been a subject of past research. ${ }^{9}$ The use of oblique shock waves to enhance mixing has been proposed, tested, and used in practice. ${ }^{10,11}$

Other studies have investigated the use of pre-injection on the vehicle forebody in order to allow more time for mixing with the goal of reducing combustor length. Rubins and Bauer ${ }^{12}$ described an early analytic treatment of pre-injection with application to scramjet engines from Mach 9 to 22 flight conditions and concluded that premixing with hydrogen has the ability to improve inlet pressure recovery, reduce friction, and shorten the vehicle combustor. A numerical study of slot-injection into a Mach 6.3 flow was conducted by Gonzalez ${ }^{13}$, investigating the parametric effect of fuel temperature and pressure on the potential for ignition in the boundary layer. Recent advances in pre-injection for the shcramjet forebody with hydrogen and hydrocarbon fuel considered the use of cantilevered injectors with slot cooling to prevent boundary layer autoignition. ${ }^{14-19}$

\section{Design Tradeoffs of Injection and Mixing}

The penetration of a transverse fuel jet into a crossflow is largely governed by the dynamic pressure ratio, $J$, of the fuel jet to the crossflow (e.g. relative force of blowing), ${ }^{2}$ the geometry of the jet itself (e.g. disturbance to the crossflow), ${ }^{7,20}$ and the angle of injection. The dynamic pressure ratio is bounded by maximum fuel system pressure and the desire to produce an underexpanded plume. An underexpanded plume is desireable because it prevents losses due to shock structures forming within the jet. The geometry of the jet is governed by the injector nozzle and its exit shape with more complex geometries representing a more difficult problem in fabrication and survivability. The angle of injection represents a design tradeoff between high-penetration/high-loss normal injection and low-penetration axial injection that imparts a beneficial thrust to the vehicle due to the fuel jet itself.

Identifying an injector that best penetrates and promotes mixing of fuel into the main flow is insufficient when considering how the injected plume is to subsequently mix with its surroundings. Molecular mixing is governed by diffusion, and how quickly fuel and air diffuse through a unit area of interface is largely out of the designer's control. Therefore, increasing the mixing rate through the fuel-air interface is accomplished by stretching the interface using vorticity in the flow. Vortical stirring structures can be induced via geometry placed in the flowfield as is the case with the swept ramp concept ${ }^{3}$ and by flow interaction phenomena 
such as that between a fuel jet passing through a shockwave. ${ }^{11}$ Care should be taken in designing such an interacting flowfield, however, as stirring phenomena can work against the designer. For example, some character of shock waves can turn fuel back toward the wall. ${ }^{14}$

Once a fuel injector is identified that has excellent penetration and mixing characteristics, the designer is once again presented with a challenge in the form of losses produced by the injector concept. For example, there may exist an injector concept that is able to achieve perfect mixing but at the cost of depleting the main flow of the momentum upon which the vehicle depends for producing thrust. There are a number of loss sources that must be taken into account when designing and implementing a forebody injector. For example, separation zones are likely to develop that may entrain the fuel from the injector and enhance local combustion. In particular this is observed in flush-wall injection. ${ }^{21}$ This is a potential source of drag from combustion on the forebody as well as fuel loss. Combustion in the vicinity of the injector also has the potential for increasing local heating rates. Another source of drag is autoignition due to the fuel entering the hot boundary layer, igniting, and pressurizing the forebody. This is a significant source of drag, fuel loss, and intense heating at the wall. This issue can be mitigated with film or transpiration cooling; however, care should be taken in this approach due to the negative impact on the specific impulse with excess fueling. Other sources of drag include intrusive geometries and unpressurized, rearward facing steps. Finally, injection schemes that deviate from axially directed injection suffer increasing momentum losses as the angle of injection approaches 90 degrees. Some of these factors may actually be a benefit in the context of internal injection (e.g. rapid autoignition) but are detrimental for forebody injection.

\section{Performance Parameters}

\section{Mixing Efficiency}

Mixing efficiency is used in this study as a measure of the effectiveness of a particular fuel injection concept to provide uniform mixing. Ideal conditions for combustion consist of uniformly distributed fuel-air at a local equivalence ratio of one at each point. Mixing efficiency is an integrated value over a given flow plane and is defined by the ratio of mixed oxidizer mass flow rate divided by the total fuel oxidizer flow rate. The definition given by Mao et al. ${ }^{22}$ is

$$
\eta_{m}=\frac{\dot{m}_{O_{2}, \text { mix }}}{\dot{m}_{O_{2}, \text { total }}}=\frac{\int_{A, Y=0} Y_{R} \rho u d A}{\int_{A, Y=0} Y \rho u d A}
$$

where

$$
Y_{R}=\left\{\begin{array}{cl}
Y & \text { for } Y \leq Y_{S} \\
\frac{1-Y}{1-Y_{s}} Y_{s} & \text { for } Y>Y_{S}
\end{array}\right.
$$

Because the mixture is globally fuel rich due to spillage of predominantly oxygen-containing flow from the open geometry, the mass fractions in these equations are that of oxygen; otherwise fuel mass fractions would be used. Mixing efficiency represents an upper bound on the combustion efficiency, which is a measure of fuel consumption rather than mixing.

\section{Thrust Potential}

The typical loss figure of merit used to evaluate forebody flows is total pressure recovery. This is due to the ease with which it can be calculated or one-dimensionalized from a multidimensional flow. The use of such a parameter as a true indicator of irreversibility generation in the forebody flow implicitly assumes a nearly constant total temperature. For forebody mixing problems, however, such an assumption breaks down due to the mixing of two streams of distinct total temperature and because flow spillage may be present. This issue is remedied by using the stream thrust potential parameter. 


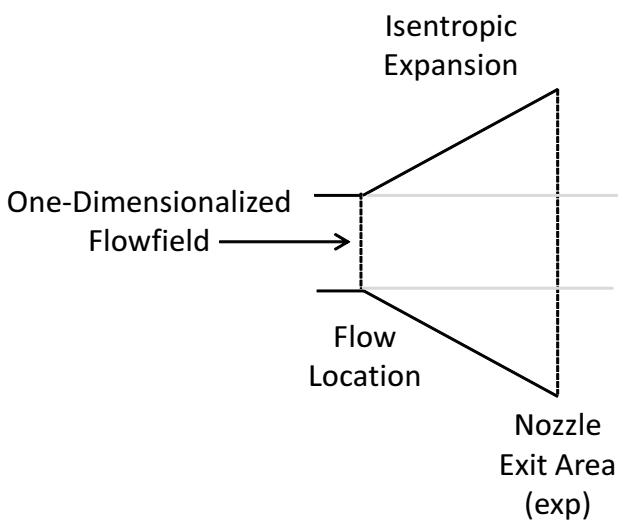

Figure 2. A schematic of the stream thrust potential methodology.

Past studies have used thrust-based methods to identify, account for, and optimize on different loss mechanisms occurring in a flowpath. ${ }^{21,23-25}$ Its usefulness has been demonstrated in the design of complete engine flowpaths. ${ }^{26}$ Although the current study only considers a single component of a full flowpath (i.e. a forebody injector), stream thrust potential still provides a good metric for comparing different injection concepts. The stream thrust potential is given by the equation

$$
F=\dot{m} u_{e x p}+p_{\exp } A_{\text {exp }}
$$

The stream thrust potential is obtained by one-dimensionalizing the flow at a given plane according to a method that conserves mass, energy, and entropy ${ }^{27}$. Once the one-dimensionalized parameters are obtained, the flow at a given flow station is expanded isentropically to a reference nozzle area, shown schematically in Figure 2. For this study the reference nozzle area is set equal to the capture area of the notional flight vehicle. Mixing, heat loss, friction, and shock waves will in general reduce stream thrust potential while heat addition and axial fuel injection will increase stream thrust potential. Hence, as with mixing efficiency, using stream thrust potential by itself as a metric for comparison is insufficient because good mixing will negatively impact stream thrust potential.

\section{Modeling and Simulation}

\section{Flow Conditions}

The simulated flow is on a flat plate at conditions representative of the forebody of an airbreathing vehicle traveling at Mach 12 at a dynamic pressure of 71,820 Pa (1500 psf). Using the 1976 US standard atmosphere, such a point on the flight trajectory occurs at an altitude of $33.33 \mathrm{~km}$. Representative flat plate conditions are obtained by processing the freestream flow through a five degree turn followed by a six degree turn. The notional forebody geometry used in this study to obtain the flat plate conditions is shown in Figure 3. Freestream and flat plate conditions are summarized in Table 1.

Each injector is sized assuming the engine flowpath captures $4.5 \mathrm{~kg} / \mathrm{s}$ of air (corresponding to a smallscale engine) at the conditions in Table 1 and that the injector is one of five on the forebody combining to provide a globally stoichiometric fuel flow rate. For the case of transverse fuel injection, sonic injection with a dynamic pressure ratio of 1.0 is ensured. For transverse injection, the fuel jet is underexpanded in order to prevent oblique shock structures from forming within the jet ${ }^{28}$. For ramp and strut injection, pressure matching between the injector exit and the inflow is assumed in an attempt to preserve vortical flow structure. An additional ramp injection case with sonic injection is simulated to evaluate the effect of 


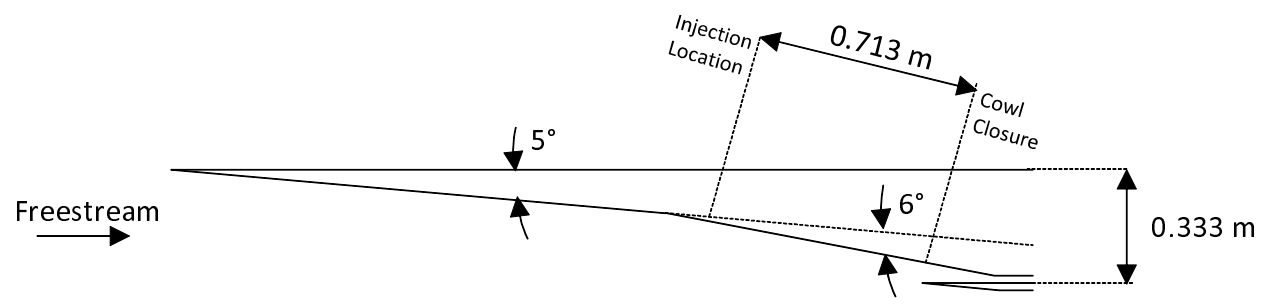

Figure 3. The baseline forebody geometry used in this study is shown.

Table 1. Freestream and flat plate conditions. Flat plate conditions are obtained by shocking the freestream flow through a 5 degree turning followed by a 6 degree turning. All cases use an air molar composition of $79 \% \mathrm{~N}_{2}$ and $21 \% \mathbf{O}_{2}$

\begin{tabular}{rcc}
\hline \hline & Freestream & Flat Plate \\
\hline Static pressure & $713 \mathrm{~Pa}$ & $9111 \mathrm{~Pa}$ \\
Static temperature & $232 \mathrm{~K}$ & $541 \mathrm{~K}$ \\
Mach number & 12.0 & 7.67 \\
\hline \hline
\end{tabular}

Table 2. Fuel injector exit conditions. All cases use $100 \% \mathrm{H}_{2}$ at a static temperature of $390 \mathrm{~K}$.

\begin{tabular}{rcccc}
\hline \hline & Transverse & Ramp (Matched) & Ramp (Sonic) & Strut (Matched) \\
\hline Injector Mach number & 1.0 & 3.92 & 1.0 & 2.60 \\
Dynamic pressure ratio & 1.0 & 0.26 & 0.07 & 0.26 \\
Static pressure & $535,000 \mathrm{~Pa}$ & $9111 \mathrm{~Pa}$ & $35,715 \mathrm{~Pa}$ & $9111 \mathrm{~Pa}$ \\
Injector exit area & $52.6 \mathrm{~mm}^{2}$ & $790 \mathrm{~mm}^{2}$ & $790 \mathrm{~mm}^{2}$ & $790 \mathrm{~mm}^{2}$ \\
\hline \hline
\end{tabular}

higher pressure on injection and mixing. In all cases it is assumed that the fuel system is able to supply the pressures required for the injection system. The assumed and derived injector conditions are summarized in Table 2 .

\section{Injector Geometries}

\section{Flush-Wall Injection}

In this study transverse injection is simulated through flush-wall nozzles of varying exit geometry. While flush wall injectors may be angled to achieve an axial component of the fuel flow, only transverse injection is considered herein in order to understand the limits of mixing obtainable by a flush wall injector. Injector concepts to be considered include a circular wall injector as well as injectors with leading edge geometrical modifications that make them more suitable for a forebody injection environment.

The flush wall nozzle geometries studied herein are depicted in Figure 4 with parameters given in Table 3. Of the four geometries considered, three are primitive shapes designed to give a basic understanding of the effect of leading- and trailing-edge bluntness and sharpness on the evolution of mixing and losses downstream of the injection location. Sharp leading edge modifications to flush-wall injectors are proposed to reduce upstream recirculation of fuel. The fourth geometry was proposed by Hirano et al. ${ }^{7}$ and has been demonstrated to produce enhanced plume area and penetration in a low Mach number cross flow. This geometry, referred to as a stinger, is evaluated to study its effect in high Mach number cross flow. 

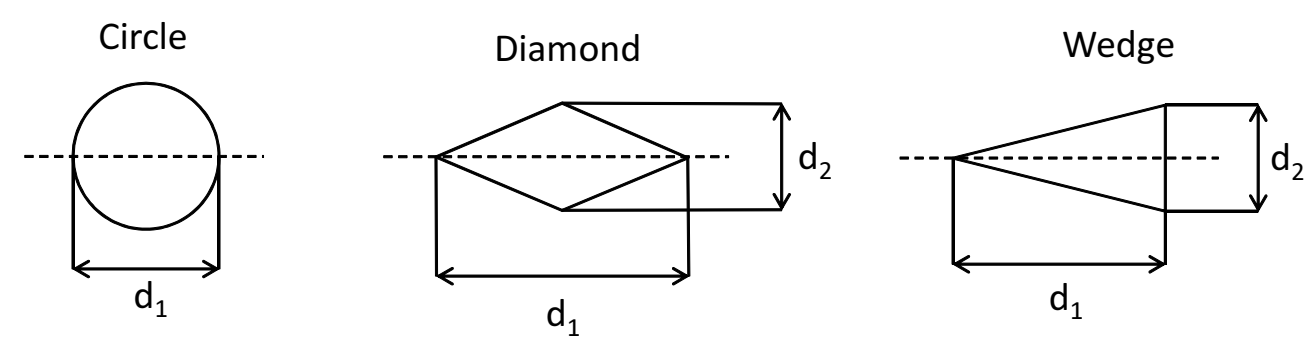

Stinger

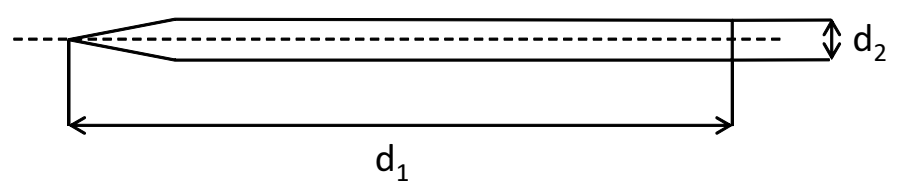

Figure 4. Notional geometry for flush wall injectors.

Table 3. Injector geometry for flush-wall injection. All injectors have the same exit area.

\begin{tabular}{rcc}
\hline \hline Geometry & $\mathbf{d}_{\mathbf{1}}(\mathrm{mm})$ & $\mathbf{d}_{\mathbf{2}}(\mathrm{mm})$ \\
\hline Circular & 8.19 & - \\
Diamond & 24.44 & 4.31 \\
Wedge & 17.28 & 6.09 \\
Stinger & 36.83 & 1.64 \\
\hline \hline
\end{tabular}

\section{Ramp Injection}

The ramp geometry has been studied and used in internal supersonic flows for the axial injection of fuel as well as inducing vortical flow structures to enhance mixing. Marble and Hendricks ${ }^{11}$ proposed an expansion ramp concept in order to enhance mixing through the interaction of the fuel plume with the shock wave emanating from the base of the ramp. This interaction induces baroclinic torque due to the unaligned density and pressure gradients at the fuel-air boundary and causes a roll-up of the fuel in the direction the shock sweeps through the fuel plume. Ramp injectors that are swept induce additional vorticity as flow rolls off of the edges of the ramp. ${ }^{3}$

In this study the expansion ramp injector of Waitz et al. ${ }^{29}$ is modified for the specific requirements of forebody fuel injection. The face of the injector base below the injection location is cut back upstream in order to eliminate the fueled recirculation region that would otherwise be observed at the base. Such a recirculation region increases the possibility of autoignition on the forebody due to the hot stagnation region behind the ramp. The geometry modification is done in two ways. The first is by simply cutting a cavity out of the base of the injector below the duct that is supplying fuel. This type of geometry has been studied by Parent et al. ${ }^{14-19}$ at similar conditions and is referred to as a cantilevered ramp injector. The second cuts back the injector base in an expansion wedge, as shown in Figure 5. This geometry is used in an attempt to increase pressurization on the base of the injector while discouraging recirculation. A summary of the cases tested are given in Table 4. 


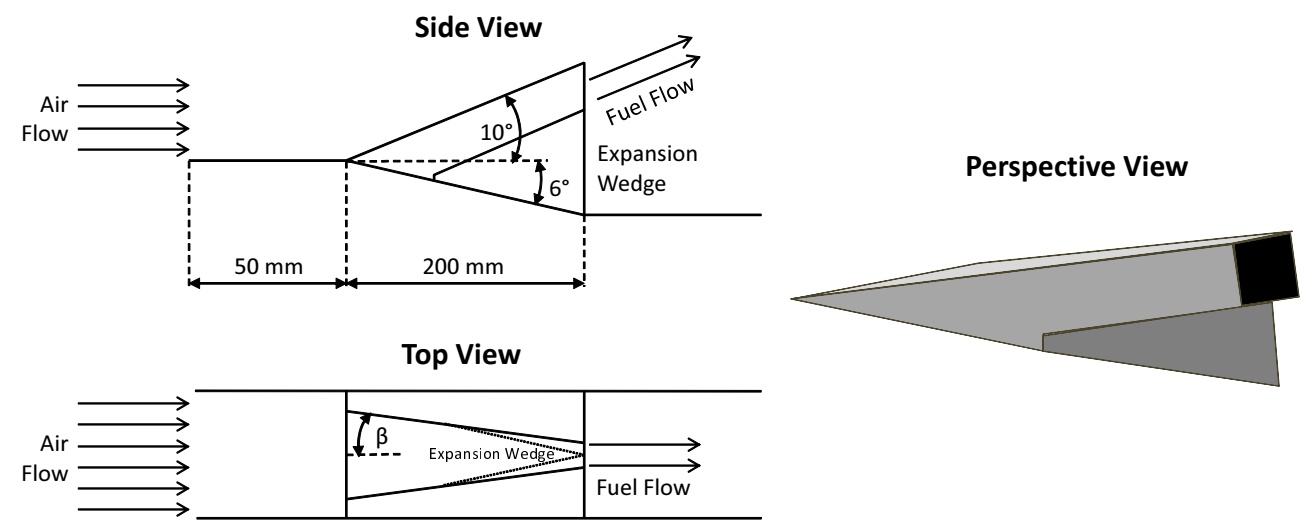

Figure 5. Side-, top-, and perspective-view geometry for modified ramp injectors.

Table 4. Modified ramp cases.

\begin{tabular}{rcc}
\hline \hline Case & $\beta(\mathrm{deg})$ & Base Wedge? \\
\hline Swept ramp (no wedge) & 3.0 & No \\
Swept ramp (with wedge) & 3.0 & Yes \\
Unswept ramp (no wedge) & 0.0 & No \\
Unswept ramp (with wedge) & 0.0 & Yes \\
\hline \hline
\end{tabular}

\section{Strut Injection}

Like the ramp injector, the strut has also been used in internal flows for supersonic fueling and mixing. The typical strut spans the entire flowpath and is characterized as a fuel placement device rather than a fuel mixing device. The strut injector may potentially be the ideal fuel injector for large-scale (e.g. orbital access) flowpaths where the ability for ramps or flush-wall injectors to access the core flow is diminished. Geometries that have heights deep into the fuel core but do not span the entire flowpath have been called either struts or pylons in the literature-for the purposes of this study they will also be called struts. Up to this point studies of forebody injection via struts have not been deeply investigated in the literature.

This study considers a single strut injector geometry that is swept back 45 degrees and injects fuel away from the injector centerline at a 10 degree angle. A schematic of the injector is shown in Figure 6. As with the ramp injector, the strut injector is placed on an expansion ramp in order to take advantage of shock-induced baroclinic torque between the fuel plume and the surrounding air. Rectangular ports supply fuel injection with expansion wedges cut between injectors in order to reduce base recirculation. The strut design proposed here is a non-optimized point design meant to be an initial investigation into forebody strut injection.

\section{Computational Domain}

The computational grids have approximately 5 - 6.5 million grid points with clustering near the injector location. For each case the mixing region of the domain has the same grid density in order to ensure a reasonable comparison between different cases. The computational domain is representative of one-half of the physical domain by using a symmetry boundary condition along the flow centerline bisecting the injector. All walls are set to be isothermal surfaces at a temperature of $500 \mathrm{~K}$. The inflow boundary is fixed at the conditions given in Table 1, representative of conditions on the second ramp of the notional Mach 

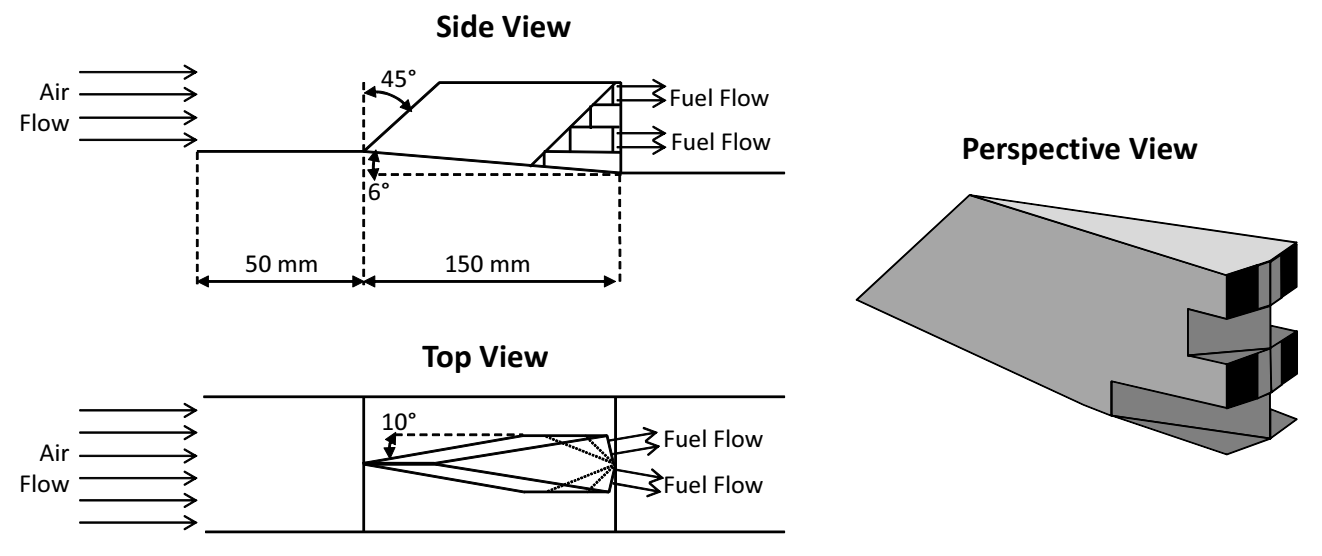

Figure 6. Side- and top-view geometry for strut injector.

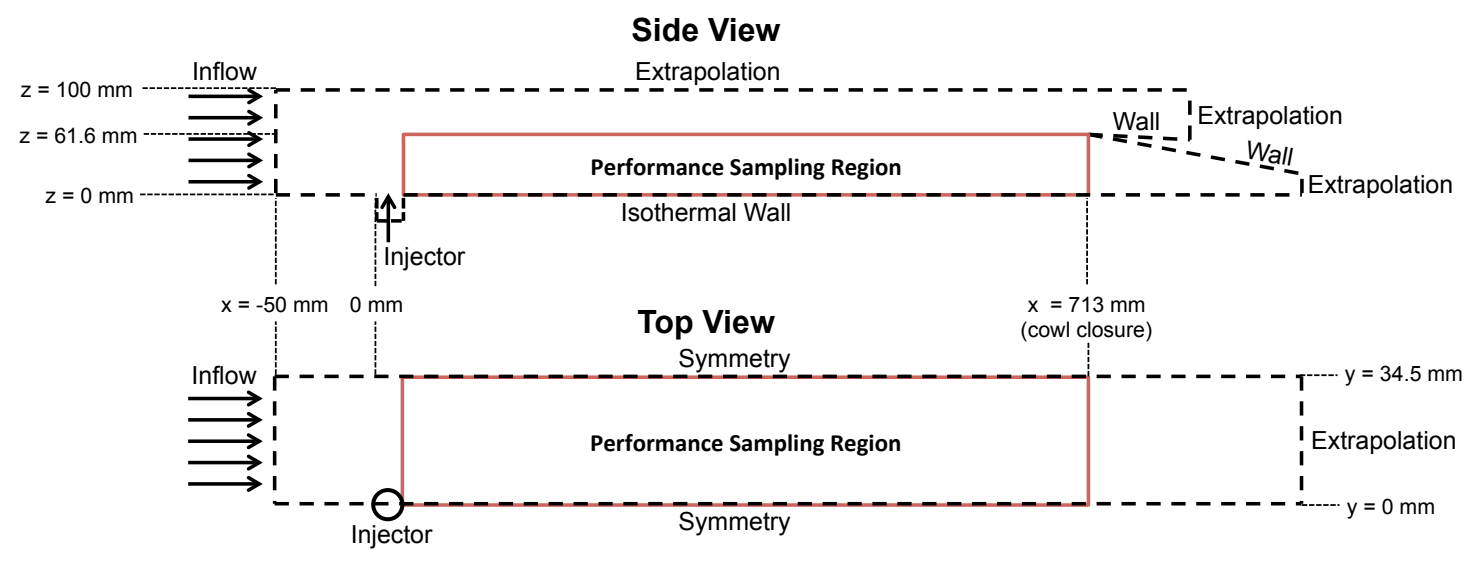

Figure 7. A side- and top-view schematic of the computational domain is shown.

12 vehicle forebody. Shocks that would otherwise appear due to forebody flow turning have been ignored for this study. A $10 \mathrm{~mm}$ portion of each fuel injection tube was simulated with slip walls; thus, the effects of boundary layer buildup and vorticity production within the injector nozzle are neglected. A schematic of a general computational domain with a flush wall injector is shown in Figure 7. The dimensions of the domain are representative of the second ramp of the Mach 12 reference vehicle. For this vehicle, there is a $50 \mathrm{~mm}$ ramp leading up to the injector, which occupies a $713 \mathrm{~mm}$ long region until cowl closure. The distance between the injector centerline and the halfway point between neighboring injectors is $34.5 \mathrm{~mm}$.

\section{Computational Method}

The analysis code used to simulate the computational domain is the Viscous Upwind aLgorithm for Complex flow ANalysis (VULCAN) ${ }^{30}$, developed and maintained at NASA Langley Research Center. VULCAN uses a finite-volume, cell-centered scheme for solving calorically- or thermally-perfect flows on a structured grid. The fluid for this study is assumed to be viscous, thermally perfect, and non-reacting. Reacting simulations and the effect of chemistry on mixing and autoignition will be addressed in future work. The flow is integrated spatially using the Edwards low dissipation flux vector split scheme (LDFSS) scheme ${ }^{31}$ to improve robustness and discontinuity resolution. The flow is integrated temporally using the diagonalized approximate factorization (DAF) scheme with global time stepping and an increasing time step schedule. 
The MUSCL interpolation scheme is third order upwind-biased with a smooth flux limiter. Turbulence calculations use the two-equation Menter k- $\omega$ model $^{32}$ with a turbulent Prandtl number of 0.9, turbulent Schmidt number of 1.0, and a turbulence intensity of 0.1 . The turbulence parameters were held constant for each simulation to ensure consistency of comparisons made between cases.

\section{Results and Discussion}

\section{Flush Wall Injection}

This subsection investigates the relative performance between different transverse wall injectors at the same fuel injection mass flow rate and injector area. Figure 8 shows fence plots of hydrogen mass fraction contours for the flush wall injectors. In general, the fuel plumes from the flush wall injectors remain close to the wall. This is undesirable when trying to prevent autoignition of the fuel in the boundary layer. Even the stinger, which exhibits the best penetration of fuel into the main flow of the four cases, maintains a fuel rich region near the wall. The circular injector has a large recirculation region upstream of the jet which increases the possibility of autoignition due to the increased residence time of entrained fuel combined with high temperatures in this region. Switching to a geometry with a sharp leading edge largely eliminates this upstream recirculation region, which is beneficial for mitigating autoignition.

Figure 9 shows the performance of each injector as a function of downstream distance for the metrics of mixing efficiency and stream thrust potential. Note that the spatial coordinate has its origin at the leading edge of each injector. The mixing efficiency curve for each injector follows a monotonically increasing trend as shown in Figure 9(a). In terms of mixing efficiency, the stinger injector is the best performer by a significant margin while the classic circular injector and the wedge injector are the worst performers at cowl closure. It is also notable that the stinger injector has the greatest fineness ratio versus the circular injector. Because each injector type has only one example of fineness ratio (i.e. injectors with the same shape but different aspect ratios were not studied), the ability to identify this as the dominant driver of mixing efficiency is limited.

Stream thrust potential as a function of distance from the injector leading edge is shown in Figure 9(b). Each of the injectors with sharp leading edges has roughly the same stream thrust potential loss with the stinger injector being the worst by a slight margin. The circular injector has a slightly better stream thrust potential compared to the sharp leading edged injectors. This may be due to its diminished mixing capacity. In general, the stream thrust potential experiences a slight decline initially due to friction and mixing. At around $\mathrm{x}=0.2 \mathrm{~m}$ the stream thrust potential experiences a sharp decline due to flow spillage from the top of the investigated domain. Because the mass flow rate is a dominant parameter in the stream thrust potential equation this causes a corresponding drop in stream thrust potential (even if the studied area and pressure remain otherwise unaffected). Therefore flow spillage is accounted for in the stream thrust potential calculation (as opposed to total pressure loss, which may be unaffected by spillage).

It is difficult to analyze mixing efficiency and stream thrust potential separately to get an idea of which injector performs the best. For example, just considering mixing efficiency, the stinger injector performs the best while stream thrust potential indicates that the circular injector is the best performer. Additional insight can be gained by considering stream thrust potential as a function of mixing efficiency, plotted in Figure 9(c). This plot shows how much stream thrust potential is recovered for a given level of mixing for each concept. This curve gives the analyst an idea of the value of each injector. Among the flush wall injectors considered, the stinger injector is the best value because for any given level of mixing it has the greatest stream thrust potential. Because it is able to achieve higher levels of mixing overall, it will be able to mix out faster than any other concept (thereby reducing the required forebody length).

Plots of fuel equivalence ratio just downstream of the injector and at the cowl closure location are shown in Figure 10. Just downstream of the injection location for each injector a vortex core forms in the fuel 

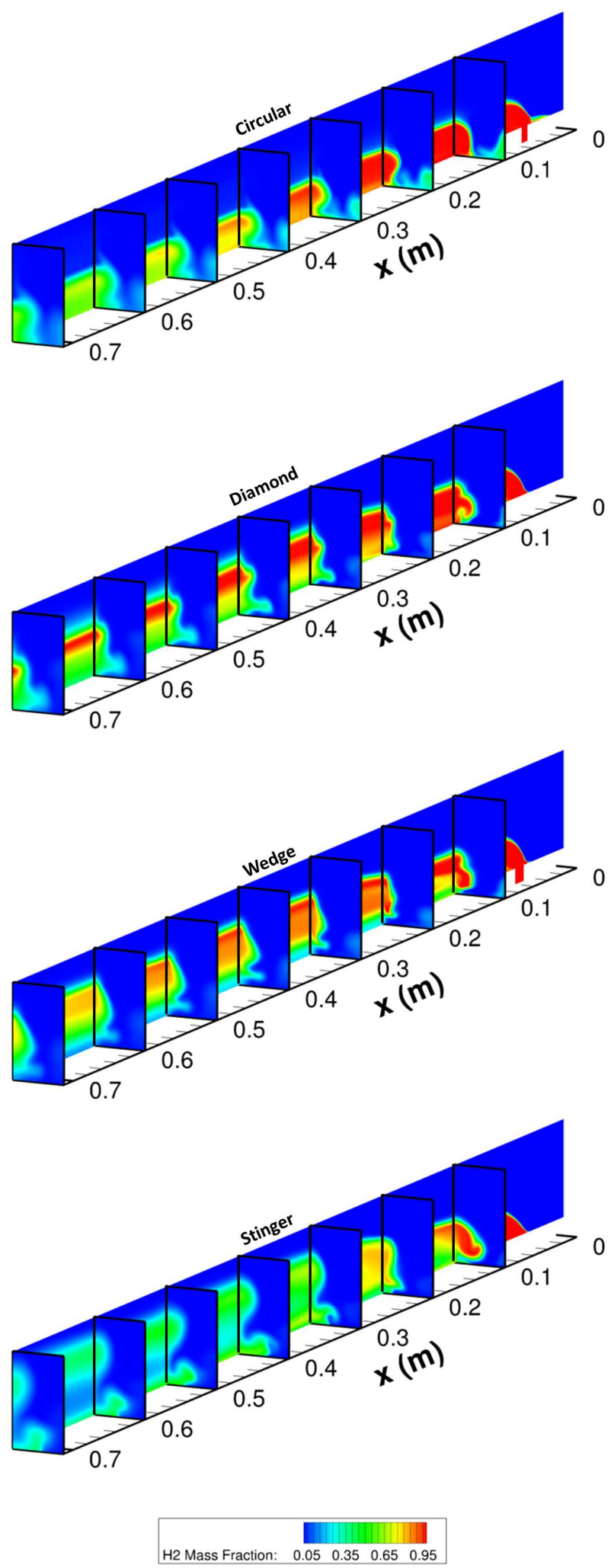

Figure 8. Fence plots of hydrogen mass fraction for the flush-wall injection cases. 


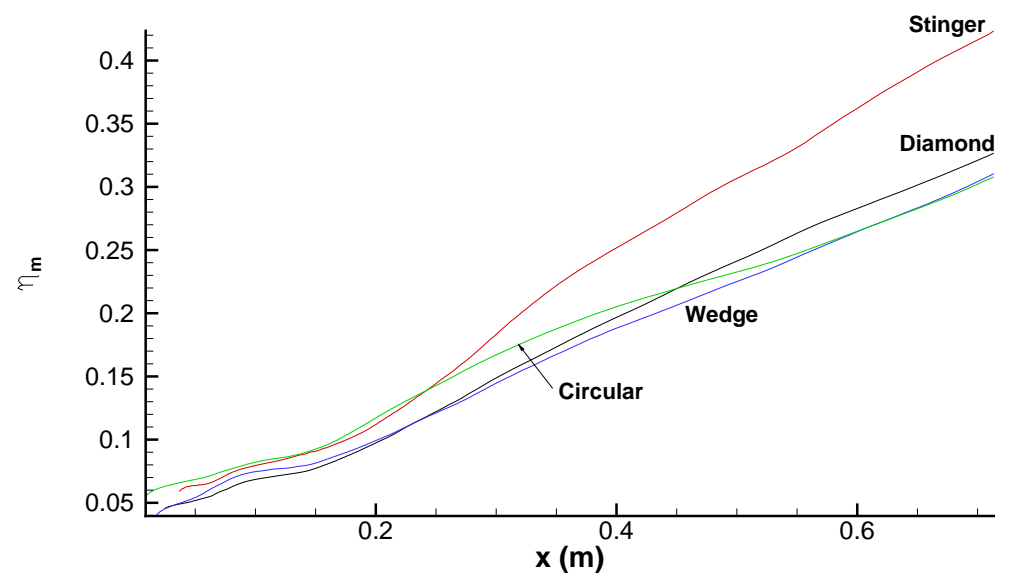

(a)

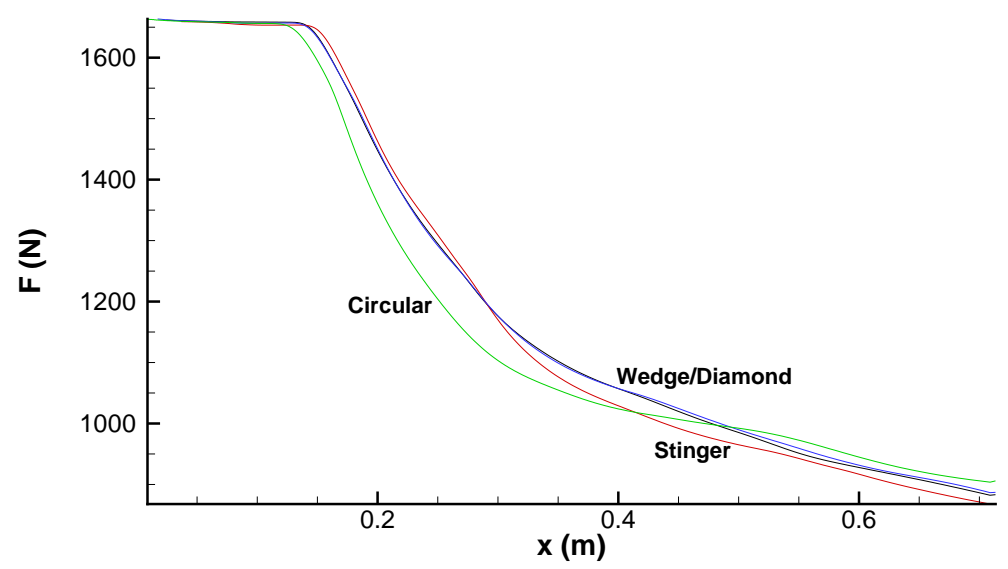

(b)

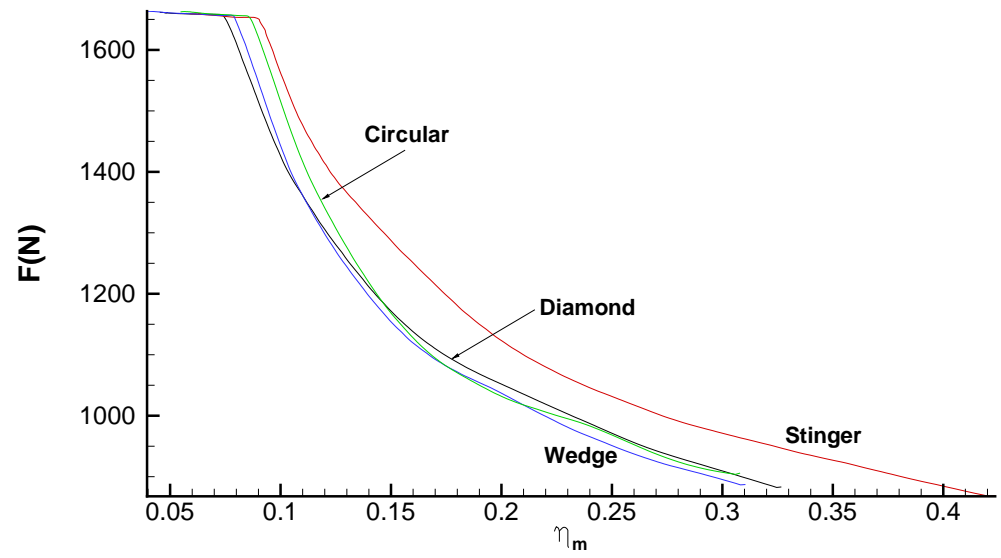

(c)

Figure 9. Performance of transverse injection concepts: (a) mixing efficiency versus $\mathbf{x}$, (b) stream thrust potential versus $\mathbf{x}$, and (c) stream thrust potential versus mixing efficiency.

\section{2 of 24}

American Institute of Aeronautics and Astronautics 


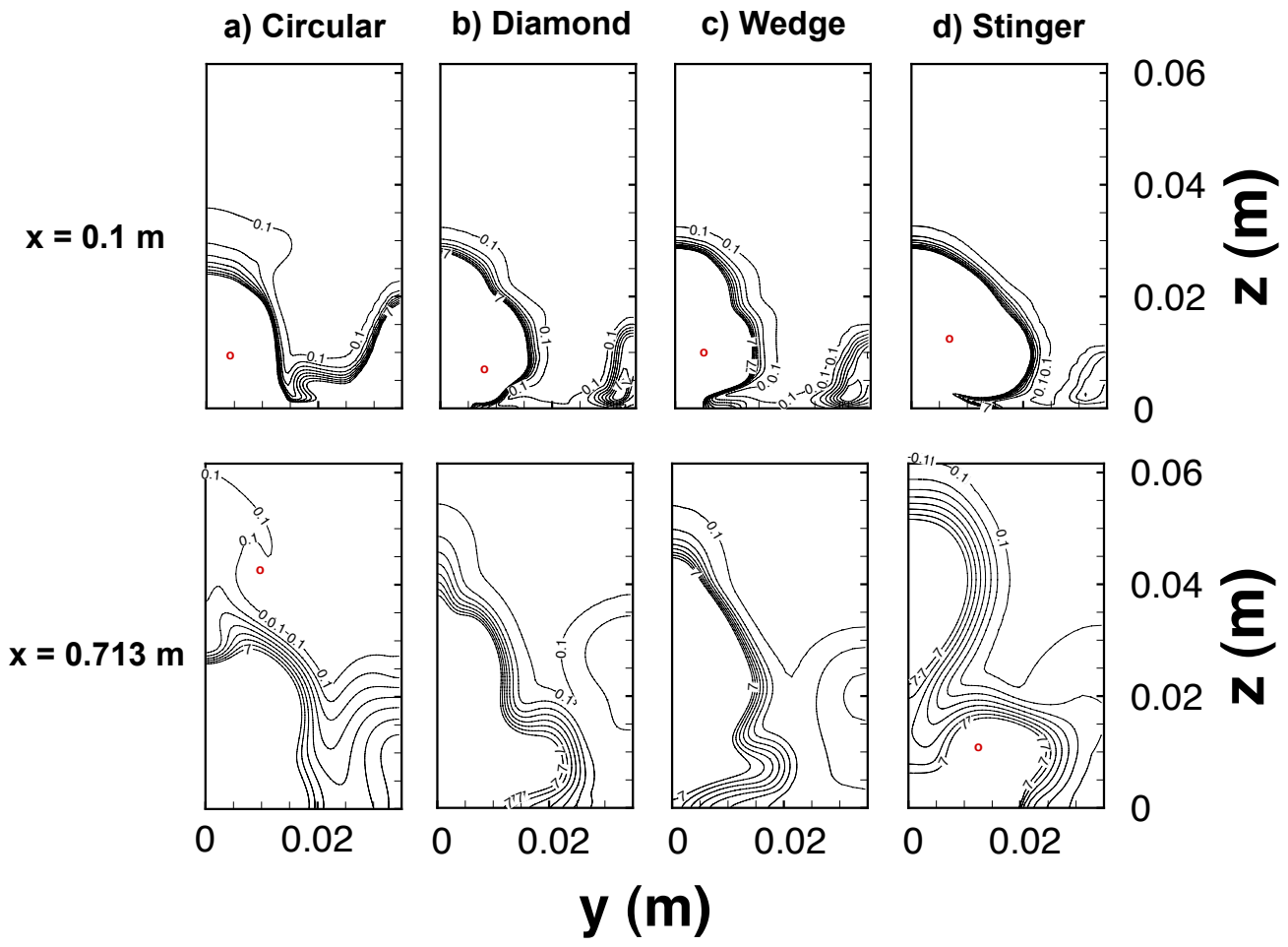

Figure 10. Fuel equivalence ratio contours for the (a) circular, $(b)$ diamond, $(c)$ wedge, and $(d)$ stinger injection case at $x=$ $0.1 \mathrm{~m}$ and $0.713 \mathrm{~m}$. Lines are plotted as $\phi=0.1,1,2 \ldots 7$. Circular marker indicates vortex center.

plume. By the time the cowl closure location in reached, however, only the circular and stinger injectors have residual vortical motion - the diamond and wedge injector plumes no longer exhibit vortical stretching of the fuel/air boundary. The vortex in the circular case, however, is relatively weak. The vorticity observed in the stinger case combined with the rising fuel bubble visible near the centerline leads to enhanced diffusivity of the fuel plume that yields its advantageous mixing performance.

\section{Ramp Injection}

This subsection assesses the performance of forebody ramp injection for the cases listed in Table 4. The objective is to assess the relative performance of ramp injectors as a consequence of varying ramp geometry and injector pressure. For each case the equivalence ratio is held constant and consideration is made toward the spillage of air from the capture streamtube. Figure 11 shows fence plots of hydrogen mass fraction contours for the ramp injector without the base expansion wedge. The pressure matched ramps, both swept and unswept, are able to inject fuel such that it mixes away from the wall. Increasing the injection pressure to a sonic condition causes the fuel to expand toward the wall and, in the case of the swept ramp, creates a recirculation region under the ramp and consequently mixes fuel near the wall.

An initial evaluation of the performance of each injector is made using stream thrust potential and mixing efficiency. Figure 12 shows the effect of geometry on the mixing efficiency and stream thrust potential for a fuel plume pressure matched to the air inflow. Geometry considerations include the sweep of the ramp and the presence of a wedge on the underside of the fuel duct. Figure 12(a) shows the mixing efficiency as a function of $\mathrm{x}$-location and begins at the injector exit. This figure demonstrates a slight disadvantage in mixing by the addition of the wedge feature and an advantage in mixing by ramp sweep. The latter result is expected due to the enhanced vorticity of the swept ramp case. 

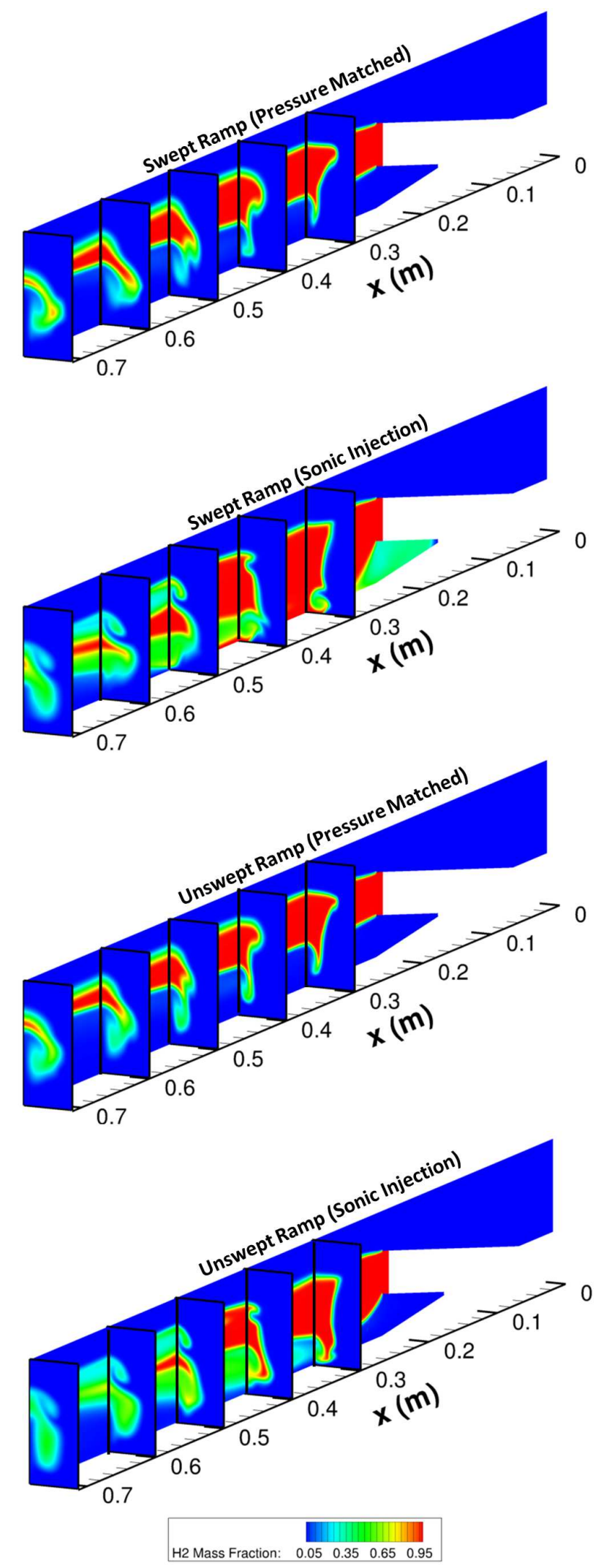

Figure 11. Fence plots of hydrogen mass fraction for the ramp injection cases without the base expansion wedge. 


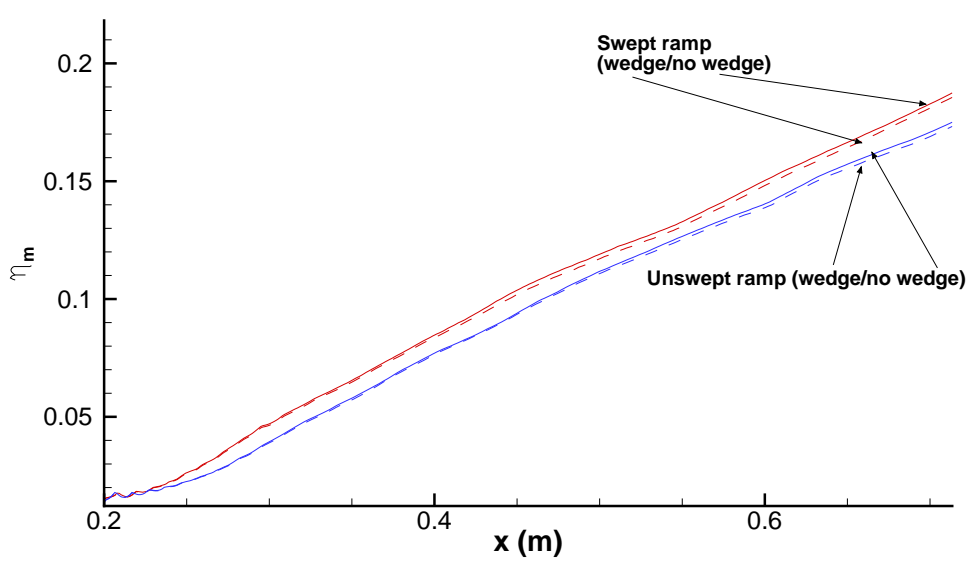

(a)

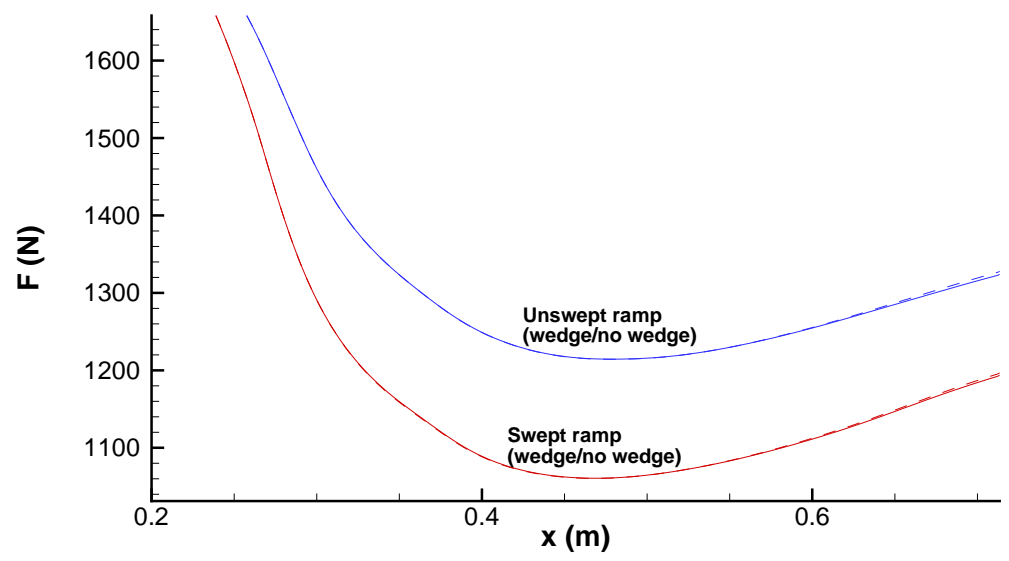

(b)

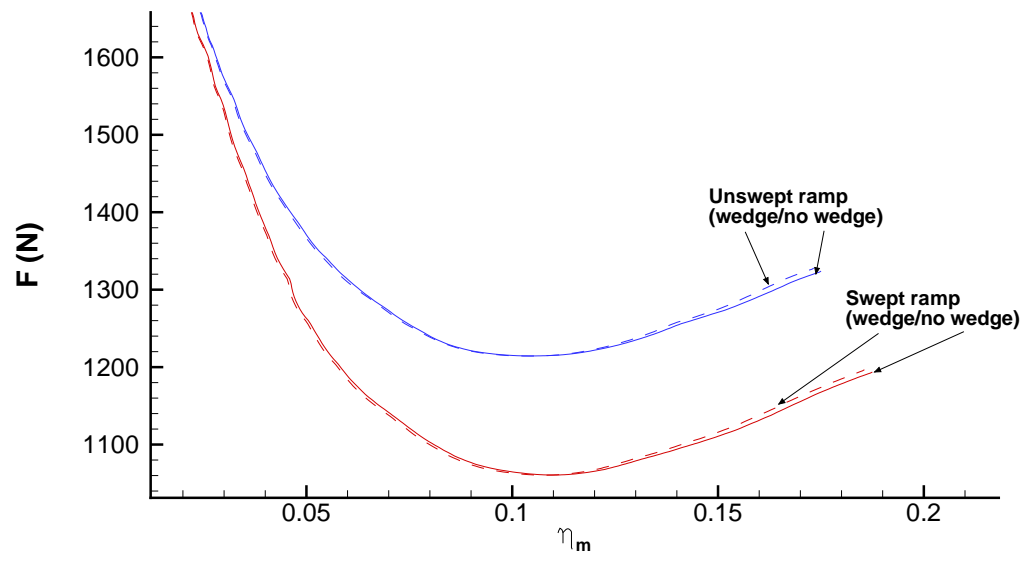

(c)

Figure 12. Performance of ramp injection concepts for matched pressure: (a) mixing efficiency versus $x$, (b) stream thrust potential versus $x$, and (c) stream thrust potential versus mixing efficiency. Dashed lines indicate cases with the expansion wedge feature.

\section{5 of 24}




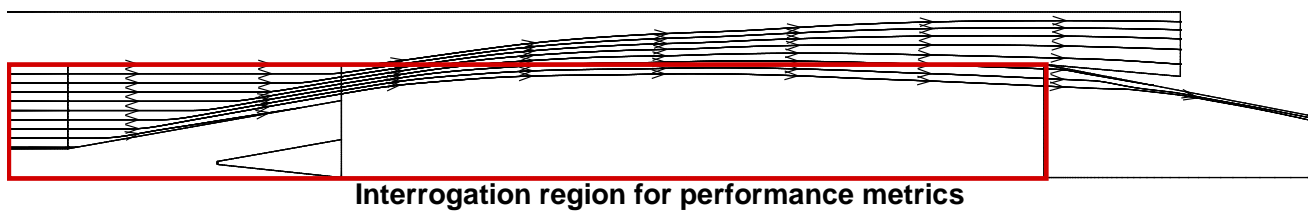

Figure 13. Stream traces that indicate flow arcing.

A larger difference in performance is observed by considering Figure 12(b), which plots stream thrust potential as a function of distance for each ramp case. Here the unswept ramp has a consistent performance benefit over the swept ramp. This is due to the fact that the wider leading edge of the swept ramp causes overall increased mass flow spillage and flow expansion due to the shock wave originating from the ramp leading edge. Eventually the flow expansion turns the downstream velocity vectors near the cowl-closure y-location back toward the body and increases the mass flow rate in the studied region. This is demonstrated by plotting centerline streamtraces in Figure 13. This causes a corresponding increase in stream thrust potential that affects each case similarly (and therefore does not substantially affect the relative performance of each concept).

Figure 12(c) plots the value of each injector concept by plotting the stream thrust potential as a function of mixing efficiency. Here it is observed that adding the expansion wedge provides a slight advantage over the cases without the feature. Furthermore, using an unswept ramp provides the best stream thrust potential for the amount of mixing obtained.

Figure 14 plots performance metrics of the ramp cases when the injector pressure is raised by a factor of 3.92 (resulting in sonic injection). Figure 14(a) shows enhanced mixing on a case-by-case basis with increased fuel pressure. This is due to the underexpanded fuel plume reaching more of the main flow and inducing additional vorticity (discussed later). At cowl closure the high pressure case has $22 \%$ better mixing relative to the pressure matched case. However, the high pressure case has lower stream thrust potential relative to the pressure matched case, as seen in Figure 14(b). Because of this, the stream thrust potential versus mixing efficiency curve in Figure 14(c) is lower than that for the pressure matched case. Therefore, among the cases considered, pressure matching is preferred-although this conclusion does not necessarily imply superiority over all other injector pressures.

A comparison of cross section slices of the flow for the pressure matched and sonic ramps are shown in Figure 15. The cross sectional slices for the cases with an expansion wedge on the injector base are substationally the same as those without, so the wedge cases are omitted here. The pressure matched cases both have the classic "kidney bean" shape at the cowl closure location that is indicative of enhanced mixing due to vorticity. The sonic injector has an additional lobe on the periphery of the fuel plume due to a secondary vortex that further enhances mixing over the pressure matched case. The pressure matched case begins to form the second vortex at an intermediate location but it is quickly absorbed by the primary vortex. The sonic injection case also has enhanced mixing near the wall which may have consequences for the possibility of boundary layer autoignition. This infiltration near the wall is due to the fact that fuel is entrained upstream of the ramp injector exit for the higher pressure case and is mixed closer to the wall due to the vorticity initiated by the ramp itself.

\section{Strut Injection}

An analysis of the performance of a forebody strut injector will now be given. Note that the discussion contained in this subsection is only for a point strut design and does not necessarily represent an optimum 


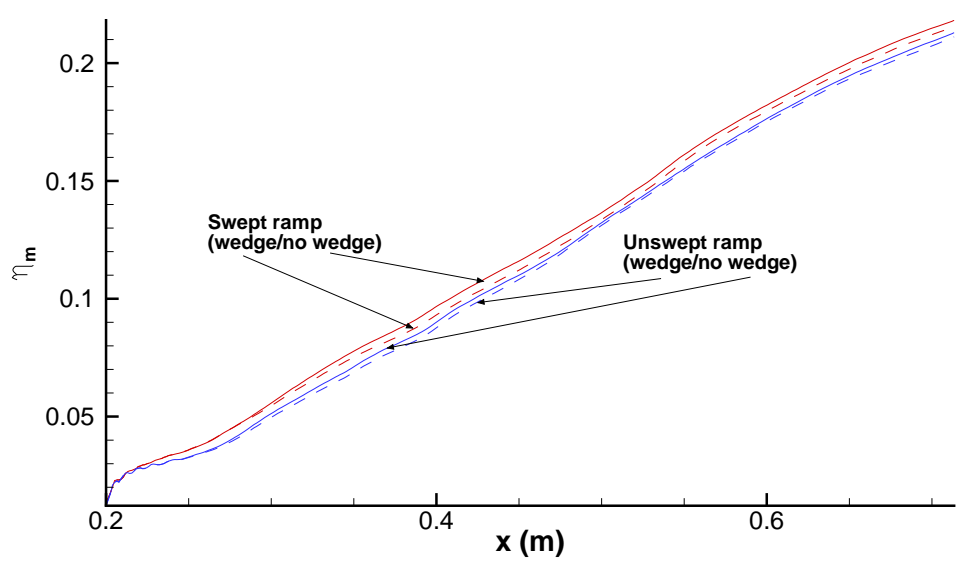

(a)

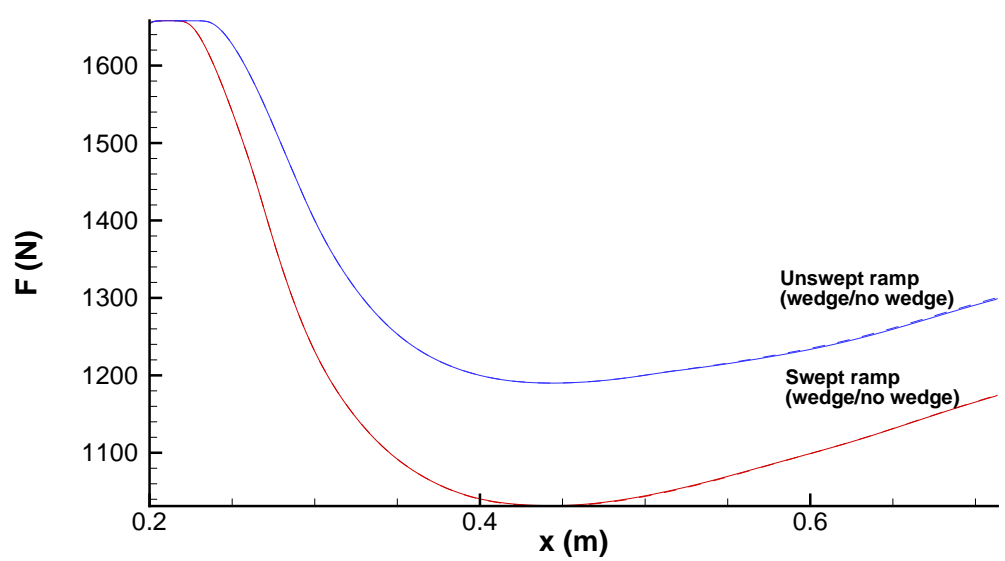

(b)

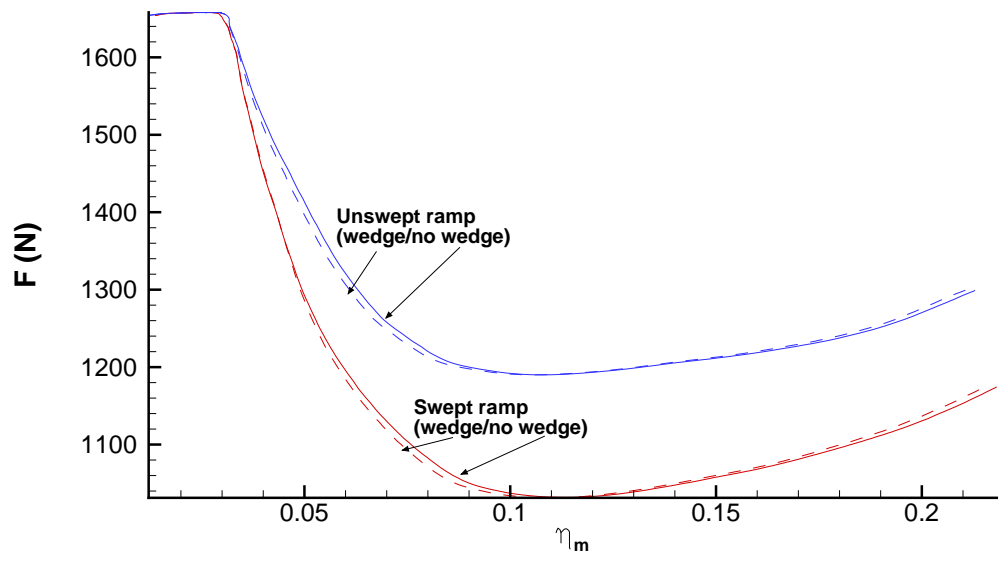

(c)

Figure 14. Performance of ramp injection concepts for sonic pressure: (a) mixing efficiency versus $x$, (b) stream thrust potential versus $x$, and (c) stream thrust potential versus mixing efficiency. Dashed lines indicate cases with the expansion wedge feature.

\section{7 of 24}

American Institute of Aeronautics and Astronautics 


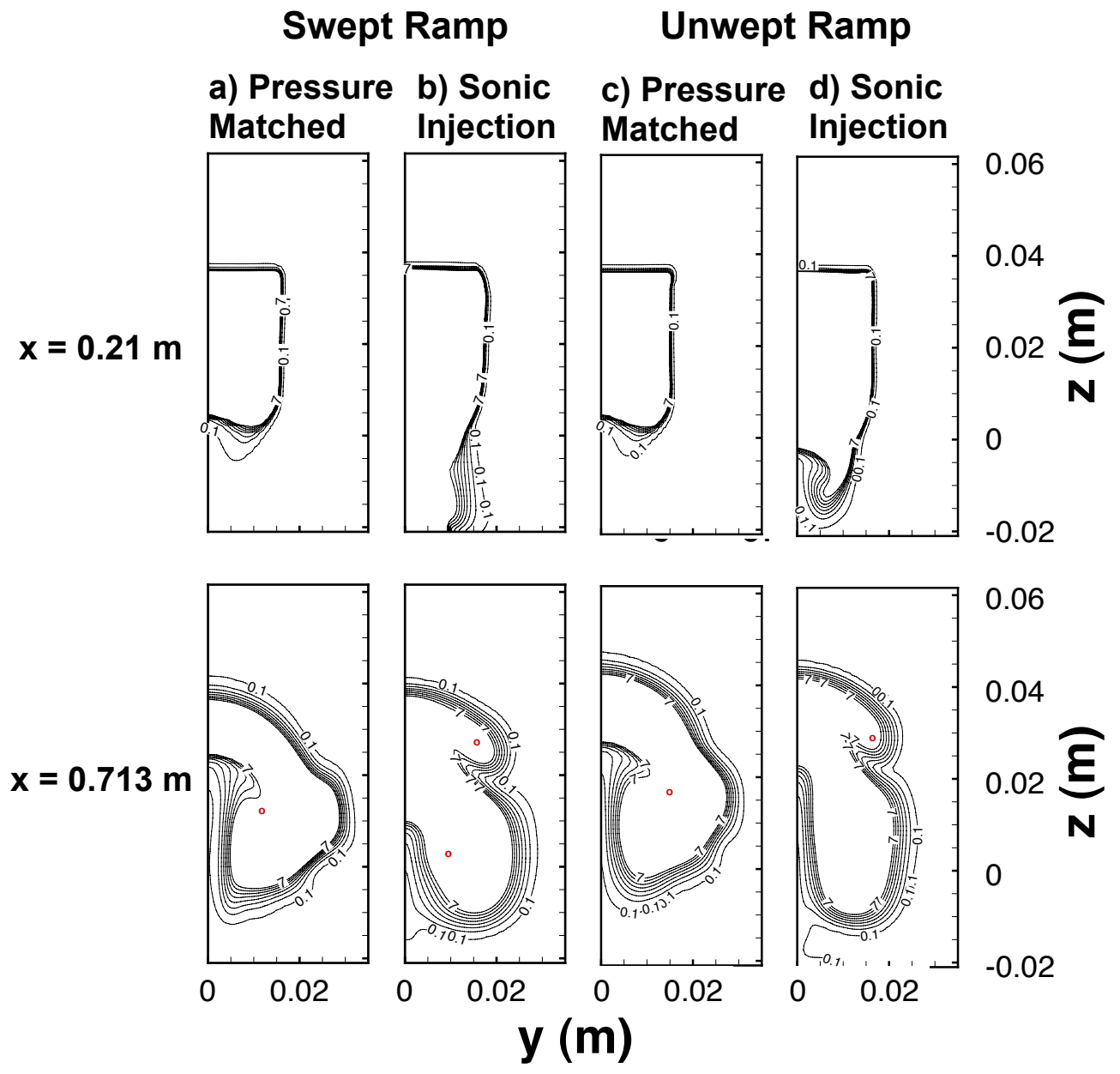

Figure 15. Fuel equivalence ratio contours for the swept and unswept ramp cases at $x=0.21 \mathrm{~m}$ and $0.713 \mathrm{~m}$. Lines are plotted as $\phi=0.1,1,2 \ldots 7$. Circular marker indicates vortex center. 


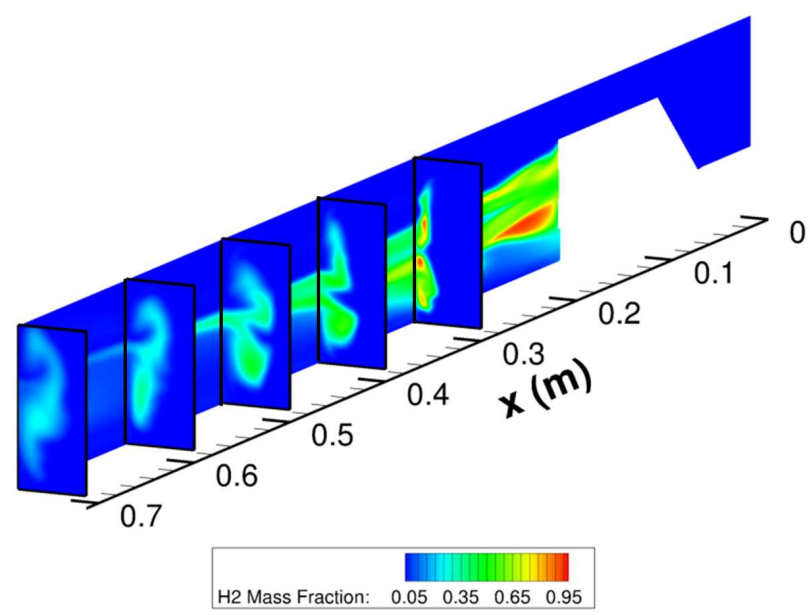

Figure 16. Fence plot of hydrogen mass fraction for the strut injection case.

configuration. Parametric studies of strut sweep angle, fuel injection angle, and fuel pressure have not been conducted for the given configuration. The purpose of this discussion is to get an idea of some of the general performance characteristics of a strut in forebody flow conditions as a starting point for future analysis.

Figure 16 shows a fence plot of hydrogen mass fraction contours for the strut injector. Overall the fuel plume has excellent mixing into the surrounding air, although there is some undesirable mixing near the wall. This could be mitigated in future strut designs by placing fueling ports farther from the wall. The effect of shock waves turning fuel toward and away from the centerline can also be observed in this figure.

Figure 17 summarizes the performance characteristics of the strut injector concept. Because the strut is a fuel placement apparatus, the flow is able to be seeded in multiple locations (four for this injector). The benefit to mixing efficiency is visible in Figure 17(a). The strut injector has significantly higher mixing efficiency at cowl closure than the ramp injector. One reason for this is due to the multiple-port characteristic of the apparatus. By having multiple ports, the contact surface between the fuel and the air is lengthened, allowing for improved mixing of fuel into air.

The stream thrust potential of the strut in Figure 17(b) follows a monotonically decreasing trend. Near the cowl closure point there is a shock wave that spills an additional amount of fuel. When considering the value of the mixing efficiency in Figure 17(c) it is evident that the strut is able to maintain a relatively high level of stream thrust potential for the mixing efficiency it achieves. The relative performance of this concept with respect to the other concepts is discussed in the next section.

Cross sections of fuel equivalence ratio for the strut injector just downstream of the trailing edge and at cowl closure are shown in Figure 18. In general, the flow for the strut case is shock-dominated, with the fuel jet turned toward and away from the centerline as shock waves reflect off of the two symmetry boundary conditions. The fuel plume mixes well overall with a smaller core than found with previous injector concepts. There is also vorticity present near the bottom of the fuel plume that further enhances mixing and is sustained through cowl closure.

\section{Injector Comparisons}

Comparison between injection strategies is conducted by selecting the best value performers from each injector category (transverse, ramp, and strut) and evaluating their relative performance. The best performers from the first two categories are the stinger and unswept ramp with expansion wedge. The strut is included for completeness. Figure 19 summarizes the performance of each top-performer. 


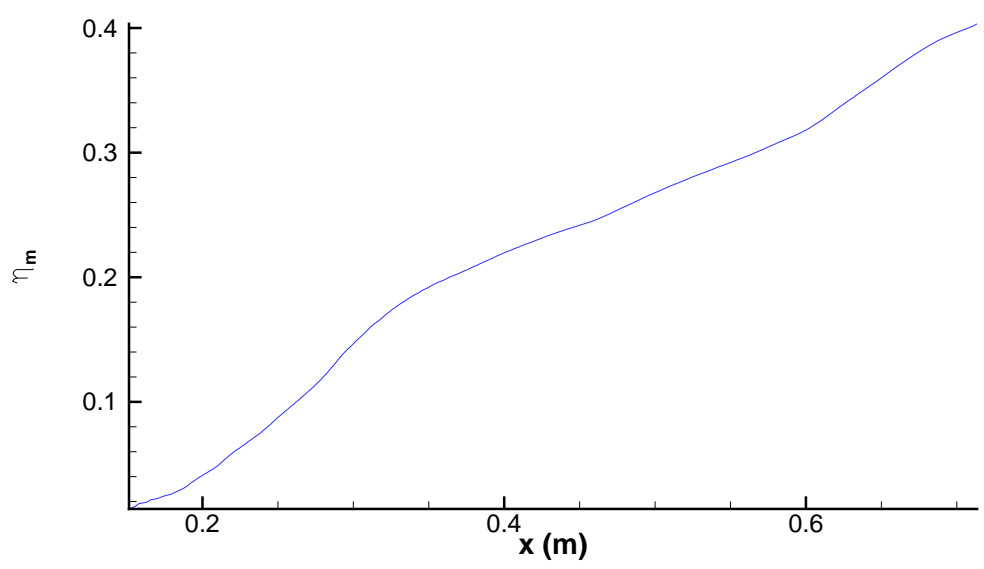

(a)

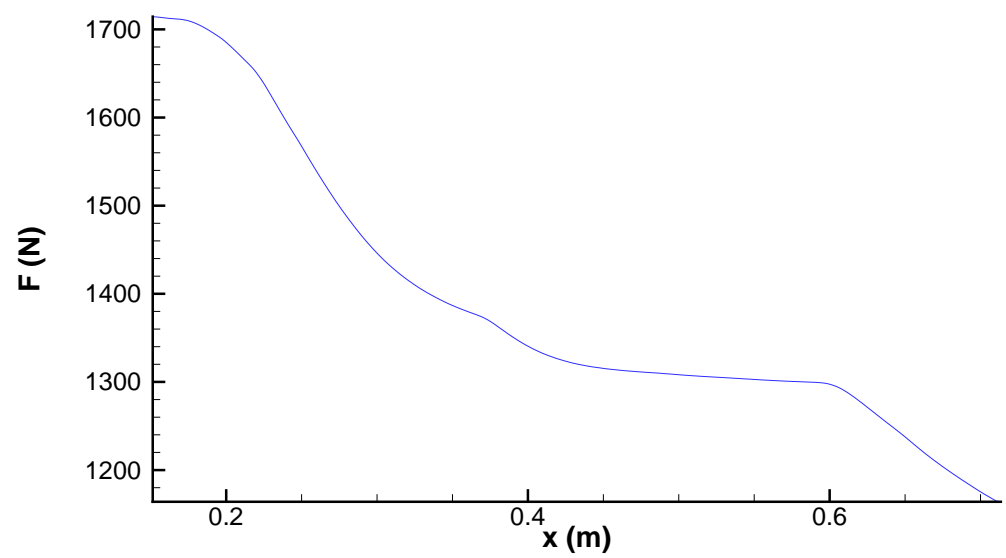

(b)

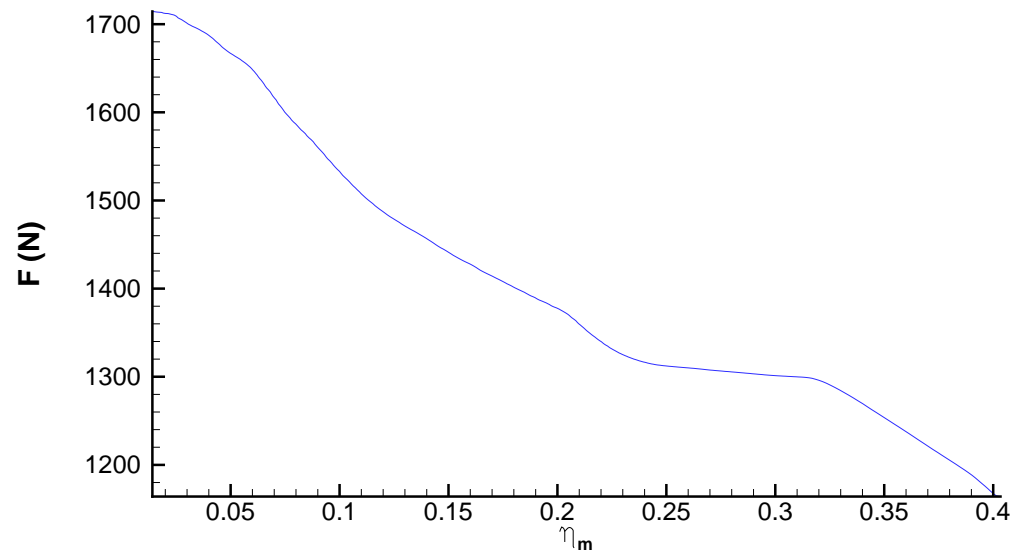

(c)

Figure 17. Performance of the strut injection concept: (a) mixing efficiency versus $x$, (b) stream thrust potential versus $x$, and (c) stream thrust potential versus mixing efficiency.

\section{0 of 24}

American Institute of Aeronautics and Astronautics 


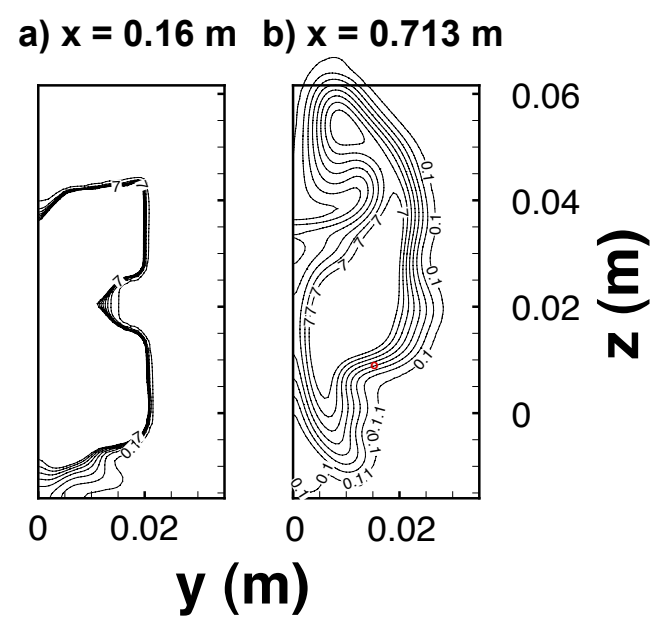

Figure 18. Fuel equivalence ratio contours for the strut injector case at $\mathbf{x}=\mathbf{0 . 1 6}$ and $0.713 \mathrm{~m} \mathbf{m}$. Lines are plotted as $\phi=0.1,1,2 \ldots 7$. Circular marker indicates vortex center.

Figure 19(a) shows the mixing efficiency as a function of location for each injector concept. While the strut injector begins to inject fuel farther downstream, it rapidly approaches the mixing efficiency achieved by the transverse stinger injector. The ramp injector has significantly lower mixing efficiency compared to the other two concepts, even considering its delayed injection location.

Figure 19(b) compares the stream thrust potential of each injector concept. The best comparison is made at the cowl closure location which represents the true captured stream tube for each concept. At this point, the ramp wedge maintains the most stream thrust potential with the transverse injector performing the poorest. This is an expected result due to the axial direction of the ramp injection and the high loss nature of transverse injection. The strut injector performs worse than the ramp injector, which may be in part due to the enhanced mixing of the strut as they both have relatively similar thrust potential at their injection locations.

Figure 19(c) plots the the stream thrust potential as a function of mixing efficiency. The location of greatest mixing efficiency for each concept is at cowl closure. The ramp injector is found to perform between the strut and transverse injectors, with the strut having the best stream thrust potential for the amount of mixing it achieves. The strut also implies a much shorter mixing length (similar to the transverse injector, only with much less stream thrust potential loss) over the ramp injector. This indicates the competitiveness of the strut injection concept and that it should be included in future forebody injection studies.

\section{Summary and Conclusions}

A numerical study has been conducted to evaluate the efficiency of different classes of injectors applied to hypervelocity forebody flow. Forebody injection was modeled by flat plate injection where the incoming conditions represented the second ramp of a hypervelocity vehicle traveling at Mach 12. All injectors have been compared using the same reference flowpath. While a flowpath tailored to a particular injector is recommended in an actual design, a single reference flowpath is adequate for initial comparisons. It was found that the forebody strut injector had the ability to provide significantly improved mixing over a forebody ramp injector while maintaining reasonable stream thrust potential levels. The transverse fuel injector, while providing the best mixing for the scale of flowpath considered here, was the worst performer when stream thrust potential was considered. The primary conclusion from this study is that the strut injector should be given additional attention as it has not been studied in the literature in the context of forebody 


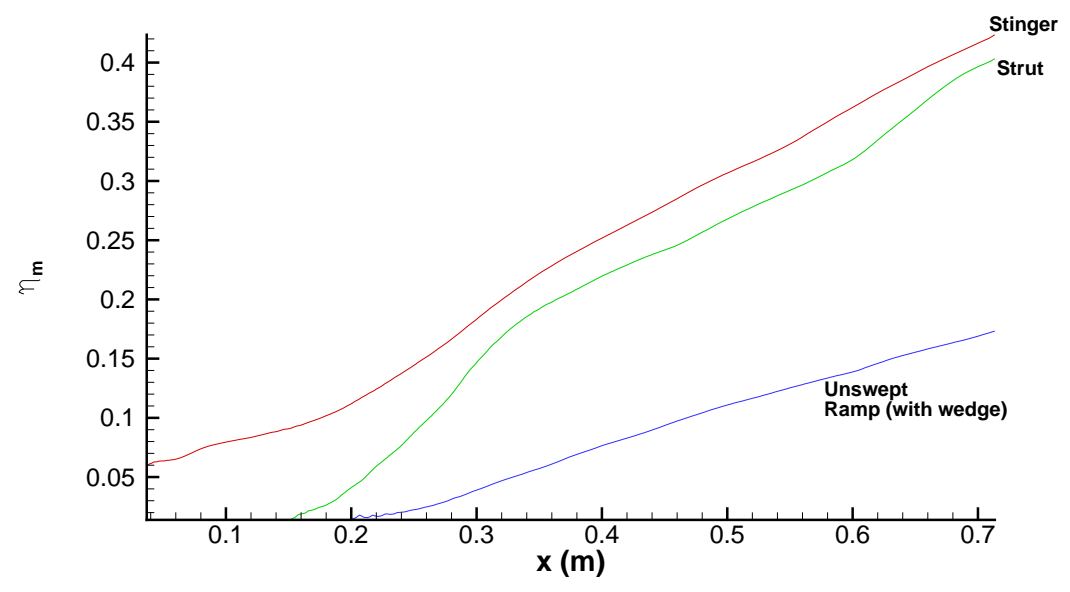

(a)

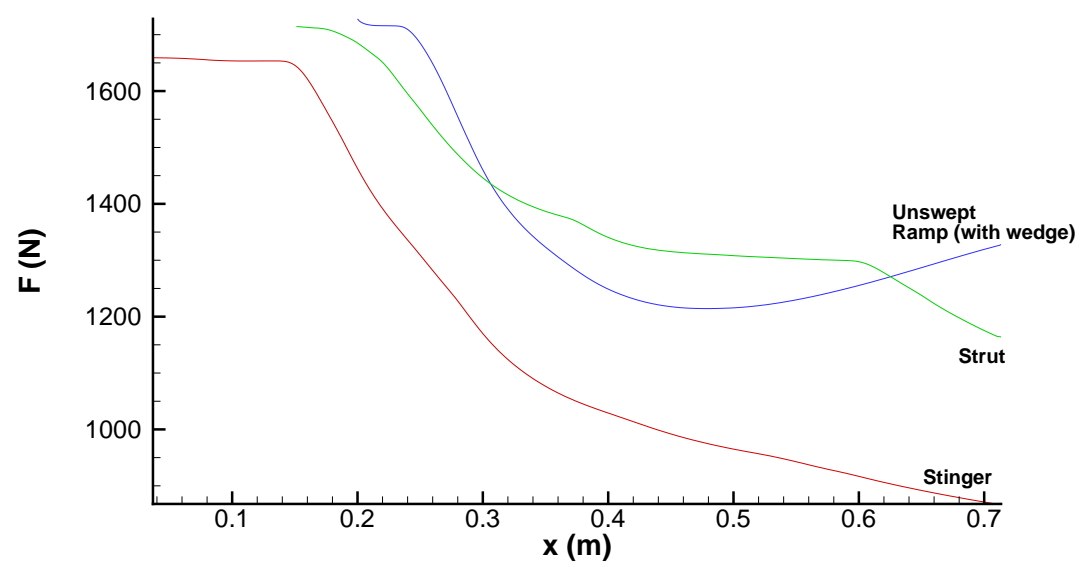

(b)

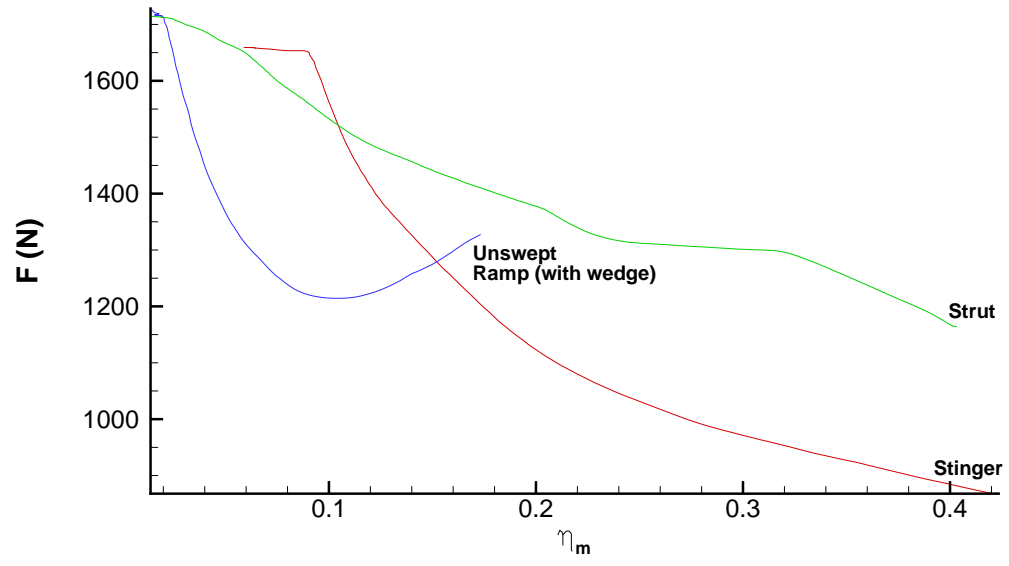

(c)

Figure 19. Performance of the best injection concepts: (a) mixing efficiency versus $x$, (b) stream thrust potential versus $x$, and (c) stream thrust potential versus mixing efficiency. 
injection.

Achieving efficient forebody fuel injection is a critical aspect of designing a successful premixed, shockinduced combustion scramjet (shcramjet) concept. While the current study only considered mixing with non-reacting fuel, understanding the conditions under which forebody autoignition may occur for a particular concept is important. Strategies for mitigation of forebody autoignition are also a necessary component of a successful forebody injection concept. Both of these requirements are avenues of work to be conducted in future studies.

\section{References}

${ }^{1}$ Axdahl, E., Kumar, A., and Wilhite, A., "Study of Unsteady, Sphere-Driven, Shock-Induced Combustion for Application to Hypervelocity Airbreathing Propulsion," in 47th Joint Propulsion Conference and Exhibit, AIAA-2011-5790, San Diego, CA, 2011.

${ }^{2}$ Billig, F., Orth, R., and Lasky, M., “A Unified Analysis Of Gaseous Jet Penetration,” AIAA Journal, Vol. 9, No. 6, 1971, pp. 1048-1058.

${ }^{3}$ Northam, G.B., Capriotti, D., Byington, C., and Greenberg, I., "Mach 2 and Mach 3 Mixing and Combustion in Scramjets," in 27th Joint Propulsion Conference, AIAA-1991-2394, Sacramento, CA, 1991.

${ }^{4}$ Northam, G.B., Greenberg, I., Byington, C., and Capriotti, D., "Evaluation of Parallel Injector Configurations for Mach 2 Combustion," Journal of Propulsion and Power, Vol. 8, No. 2, 1992, pp. 491-499.

${ }^{5}$ Byington, C.S., Northam, G.B., and Capriotti, D.P., "Transpiration Cooling in the Locality of a Transverse Fuel Jet for Supersonic Combustors," in 26th Joint Propulsion Conference, AIAA-1990-2341, Orlando, FL, 1990.

${ }^{6}$ Haimovitch, Y., Gartenberg, E., Jr., A.S.R., and Northam, G.B., "Effects of Internal Nozzle Geometry on CompressionRamp Mixing in Supersonic Flow,” AIAA Journal, Vol. 35, No. 4, 1997, pp. 663-670.

${ }^{7}$ Hirano, K., Matsuo, A., Kouchi, T., Izumikawa, M., and Tomioka, S., "New Injector Geometry for Penetration Enhancement of Perpendicular Jet into Supersonic Flow," in 43rd Joint Propulsion Conference and Exhibit, AIAA-2007-5028, Cincinnati, OH, 2007.

${ }^{8}$ Gutmark, E., Schadow, K., and Wilson, K., "Noncircular Jet Dynamics in Supersonic Combustion," Journal of Propulsion and Power, Vol. 5, No. 5, 1989, pp. 529-533.

${ }^{9}$ Quinn, J.E., Cutler, A.D., and Northam, G., "Drag Reduction of Supersonic Cavities via Mass Injection with Applications to Scramjets," in 35th Aerospace Sciences Meeting and Exhibit, AIAA-1997-0550, Reno, NV, 1997.

${ }^{10}$ Drummond, J.P., "Mixing Enhancement of Reacting Parallel Fuel Jets in a Supersonic Combustor," in 27th Joint Propulsion Conference, AIAA-1991-1914, Sacramento, CA, 1991.

${ }^{11}$ Marble, F.E. and Hendricks, G.J., "Progress Toward Shock Enhancement of Supersonic Combustion Processes," in 23rd Joint Propulsion Conference, AIAA-1987-1880, San Diego, CA, 1987.

${ }^{12}$ Rubins, P. and Bauer, R., "A Hypersonic Ramjet Analysis with Premixed Fuel Combustion,” in 2nd Propulsion Joint Specialist Conference, AIAA-1966-648, Colorado Springs, CO, 1966.

${ }^{13}$ Gonzalez, D., "Computational Study of Inlet Injection for Pre-Mixed, Shock-Induced Combustion," in 7th International Space Planes and Hypersonic Systems and Technologies Conference, AIAA-1996-4560, Norfolk, VA, 1996.

${ }^{14}$ Alexander, D.C., Sislian, J.P., and Parent, B., "Hypervelocity Fuel/Air Mixing in Mixed-Compression Inlets of Shcramjets," AIAA Journal, Vol. 44, No. 10, 2006, pp. 2145-2155.

${ }^{15}$ Schwartzentruber, T.E., Sislian, J.P., and Parent, B., "Suppresion of Premature Ignition in the Pre-Mixed Inlet Flow of a Shcramjet," in 39th Joint Propulsion Conference and Exhibit, AIAA-2003-5187, Huntsville, AL, 2003.

${ }^{16}$ Sislian, J.P., Martens, R.P., Schwartzentruber, T.E., and Parent, B., "Numerical Simulation of a Real Shcramjet Flowfield," Journal of Propulsion and Power, Vol. 22, No. 5, 2006, pp. 1039-1048.

${ }^{17}$ Sislian, J.P. and Parent, B., "Hypervelocity Fuel/Air Mixing in a Shcramjet Inlet," Journal of Propulsion and Power, Vol. 20, No. 2, 2004, pp. 263-272.

${ }^{18}$ Sislian, J., Schirmer, H., Dudebout, R., and Schumacher, J., "Propulsive Performance of Hypersonic Oblique Detonation Wave and Shock-Induced Combustion Ramjets," Journal of Propulsion and Power, Vol. 17, No. 3, 2001, pp. 599-604.

${ }^{19}$ Dudebout, R., Sislian, J.P., and Oppitz, R., "Numerical Simulation of Hypersonic Shock-Induced Combustion Ramjets," Journal of Propulsion and Power, Vol. 14, No. 6, 1998, pp. 869-879.

${ }^{20}$ Kouchi, T., Hirano, K., Matsuo, A., Kobayashi, K., Tomioka, S., and Izumikawa, M., "Combustion Performance of Supersonic Combustor with Stinger-Shaped Fuel Injector," in 44th Joint Propulsion Conference and Exhibit, AIAA-2008-4503, Hartford, CT, 2008. 
${ }^{21}$ Riggins, D., McClinton, C., Rogers, R., and Bittner, R., “A Comparative Study of Scramjet Injection Strategies for High Mach Number Flows," in 28th Joint Propulsion Conference and Exhibit, AIAA-1992-3287, Nashville, TN, 1992.

${ }^{22}$ Mao, M., Riggins, D.W., and McClinton, C.R., "Numerical Simulation of Transverse Fuel Injection," in NASA Lewis Research Center Computational Fluid Dynamics Symposium on Aeropropulsion, NASA-91N21097, Cleveland, OH, 1991, pp. 635667.

${ }^{23}$ Riggins, D., "High-Speed Engine/Component Performance Assessment Using Exergy and Thrust-Based Methods," Tech. Rep. NASA-CR-198271, NASA, 1996.

${ }^{24}$ Riggins, D., McClinton, C., and Vitt, P., “Thrust Losses in Hypersonic Engines Part 1: Methodology," Journal of Propulsion and Power, Vol. 13, No. 2, 1997, pp. 281-287.

${ }^{25}$ Riggins, D., "Thrust Losses in Hypersonic Engines Part 2: Applications," Journal of Propulsion and Power, Vol. 13, No. 2 , 1997, pp. 288-295.

${ }^{26}$ Riggins, D., "Evaluation of Performance Loss Methods for High-Speed Engines and Engine Components," Journal of Propulsion and Power, Vol. 13, No. 2, 1997, pp. 296-304.

${ }^{27}$ R.A.Baurle and Gaffney, R., "Extraction of One-Dimensional Flow Properties from Multidimensional Data Sets," Journal of Propulsion and Power, Vol. 24, No. 4, 2008, pp. 704-714.

${ }^{28}$ Schetz, J.A. and Favin, S., "An Analysis of the Ignition of Slot-Injected Gaseous Hydrogen in a Supersonic Air Stream," Combustion and Flame, Vol. 11, No. 5, 1967, pp. 397-407.

${ }^{29}$ Waitz, I., Marble, F., and Zukoski, E., "Vorticity Generation by Contoured Wall Injection," in 28th Joint Propulsion Conference and Exhibit, AIAA-1992-3550, Nashville, TN, 1992.

${ }^{30}$ NASA Langley Research Center, "VULCAN Ver. 6.1.1,” http://vulcan-cfd.larc.nasa.gov, 2010.

${ }^{31}$ Edwards, J.R., "A Low-Diffusion Flux-Splitting Scheme for Navier-Stokes Calculations," Computers \& Fluids, Vol. 26, No. 6, 1997, pp. 635-659.

${ }^{32}$ Menter, F.R., "Zonal Two Equation $k$ - $\omega$ Turbulence Models for Aerodynamic Flows," in 24th Fluid Dynamics Conference, AIAA-1993-2906, Orlando, FL, 1993. 